PHYSICAL REVIEW A 95, 059904(E) (2017)

\title{
Erratum: Analytic form of the correlation energy of the uniform electron gas [Phys. Rev. A 79, 014103 (2009)]
}

Emil Proynov and Jing Kong

(Received 24 March 2017; published 11 May 2017)

DOI: 10.1103/PhysRevA.95.059904

Two misprints in this paper were recently found that need to be corrected because they prevent reproducing the published energy results in the spin-polarized case of the uniform electron gas, which themselves are correct:

(i) Equation (23) on p. 2 should read

$$
s\left(r_{s}, \xi\right)=1.28 f_{r}\left(r_{s}\right) f_{s}(\xi)
$$

In the original paper the prefactor 1.28 was omitted.

(ii) The coefficient $a_{x}$ in Eq. (15) of the paper should read: $a_{x}=\left(3 \pi^{2}\right)^{1 / 3}$ and not: $a_{x}=\left(3 \pi^{2} / 2\right)^{1 / 3}$ as was mistyped in the paper.

The authors would like to thank Dr. M. Marques from University of Lyon1, France for pointing these misprints out to us. 